\title{
A double-blind, placebo-controlled, randomised crossover study to determine the effects of a prebiotic, a probiotic and a synbiotic upon the gut microbiota and immune response of healthy volunteers
}

\author{
C. E. Childs, P. Yaqoob, G. R. Gibson and R. A. Rastall \\ Department of Food Biosciences, University of Reading, RG6 6AP, UK
}

\begin{abstract}
Human studies have demonstrated that dietary xylooligosaccharides significantly increase the number of Bifidobacterium in faeces ${ }^{(1,2)}$. Use of Bifidobacterium as a probiotic dietary supplement has identified health benefits which include immunostimulation ${ }^{(3)}$. The primary objective of the current study was to identify the effects of xylooligosaccharides, provided as either a prebiotic or synbiotic dietary intervention, on the gut microbiota and the production of microbial metabolites, such as short-chain fatty acids. In addition, we are investigating effects on bowel function and immune function. The markers of immune function under investigation include: faecal and salivary IgA, total peripheral leucocyte numbers and phenotypes, the expression of CD69 on T-cells to in response to ConA, production of cytokines by whole blood cultures in response to LPS and ConA, plasma chemokines, and phagocytosis and oxidative burst by monocytes and granulocytes.

Forty-four healthy male and female volunteers (aged 25-65) were recruited to this double-blind study, which provides four different treatments in a crossover design of 21 days treatment and 28 days washout. The treatments under study are a prebiotic (xylooligosaccharide, $8 \mathrm{~g} / \mathrm{d}$ ), a probiotic (Bifidobacterium lactis $\mathrm{Bi}-07,10^{9} \mathrm{CFU} / \mathrm{d}$ ), a synbiotic (xylooligosaccharide $8 \mathrm{~g} / \mathrm{d}+\mathrm{B}$. lactis $\mathrm{Bi}-07$ 10 ${ }^{9} \mathrm{CFU} /$ d) and a placebo (maltodextrin).

Data indicate significant effects of the synbiotic on both the gut microflora and markers of immune function. The synbiotic increased faecal Bifidobacteria content $(P=0.046$ compared to placebo, $\mathrm{df}=34)$. There was a significant treatment effect on the percentage change in IL-10 production by LPS-stimulated whole blood cultures $(P=0.037, \mathrm{df}=32)$, with reduced IL-10 production among volunteers on the synbiotic. A trend was observed for the synbiotic treatment to increase the percentage of monocytes undergoing oxidative burst among the oldest quartile of volunteers $(P=0.075, \mathrm{df}=48)$. This indicates that the synbiotic under study has the capacity to significantly alter the gut microflora and the function of peripheral monocytes, with potential benefits to health.
\end{abstract}

This study was sponsored by Danisco Finland (Sokeritehtaantie 20, 02460 Kantvik, Finland).

1. Okazaki M, Fujikawa S \& Matsumoto N (1990) Bifidobact Microfl 9, 77-86.

2. Chung Y-C, Hus C-K, Ko C-Y et al. (2007) Nutr Res 27, 756-761.

3. Gibson GR \& Roberfroid MB (1995) J Nutr 125, 1401-1412. 